\title{
The Blood Compatibilities of Blood Purification Membranes and Other Materials Developed in Japan
}

\author{
Takaya Abe, ${ }^{1}$ Karen Kato, ${ }^{1}$ Tomoaki Fujioka, ${ }^{1}$ and Tadao Akizawa ${ }^{2}$ \\ ${ }^{1}$ Department of Blood Purification Therapy \& Urology, Iwate Medical University, School of Medicine, Iwate, Japan \\ ${ }^{2}$ Division of Nephrology, Department of Medicine, Showa University, School of Medicine, Tokyo, Japan
}

Correspondence should be addressed to Takaya Abe, takayabe@iwate-med.ac.jp

Received 11 April 2011; Accepted 25 May 2011

Academic Editor: Yukihiko Nosé

Copyright () 2011 Takaya Abe et al. This is an open access article distributed under the Creative Commons Attribution License, which permits unrestricted use, distribution, and reproduction in any medium, provided the original work is properly cited.

\begin{abstract}
The biocompatibilities in blood purification therapy are defined as "a concept to stipulate safety of blood purification therapy by an index based on interaction in the body arising from blood purification therapy itself." The biocompatibilities are associated with not only materials to be used but also many factors such as sterilization method and eluted substance. It is often evaluated based on impacts on cellular pathways and on humoral pathways. Since the biocompatibilities of blood purification therapy in particular hemodialysis are not just a prognostic factor for dialysis patients but a contributory factor for long-term complications, it should be considered with adequate attention. It is important that blood purification therapy should be performed by consistently evaluating not only risks associated with these biocompatibilities but also the other advantages obtained from treatments. In this paper, the biocompatibilities of membrane and adsorption material based on Japanese original which are used for blood purification therapy are described.
\end{abstract}

\section{Introduction}

The biocompatibilities in blood purification therapy are defined as "a concept to stipulate safety of blood purification therapy by an index based on interaction in the body arising from blood purification therapy itself." The biocompatibilities are associated with not only materials to be used but also sterilization method, eluted substance, medical agents such as anticoagulant, dialysate solution, and contamination, and so on. It is often evaluated based on impacts on cellular pathways such as leukocyte and platelet as well as on humoral pathways such as complement system, coagulation/fibrinolysis system, kallikrein-kinin system, and cytokine. For hemodialysis the biocompatibilities on blood purification therapy are not just a prognostic factor for dialysis patients but a contributory factor for long-term complications such as immunodeficiency, cardiovascular disease, and dialysis-related amyloidosis (DRA). The material of dialyzer is roughly classified into cellulose type membrane such as cellulose triacetate (CTA) membrane and synthetic type membrane including polyethersulfone (PES) membrane, polymethylmethacrylate (PMMA) membrane, ethylene vinyl alcohol (EVAL) membrane, and vitamin Ecoated polysulfone (PS) membrane.

In this paper, the biocompatibilities of membrane for dialysis and adsorption material for blood purification therapy which were developed in Japan are described.

\section{Cellulose-Type Dialyzer}

2.1. Cellulose Triacetate (CTA) Membrane. Cellulose acetate membrane is a kind of cellulose-type membrane which is synthesized by a reaction of natural polymer cellulose and acetic acid, having properties of higher transparency and toughness among thermoplastics. Cellulose diacetate is formed by substituting two hydroxyls within cellulose, and CTA is formed by substituting three hydroxyls (Figure 1). As the number of substitutions increases, hydrophilic property of the membrane decreases and complement activation of the membrane also decreases, as a result biocompatibility of the membrane improves.

This membrane is characterized by the thickness of $15 \mu \mathrm{m}$, the thinnest among commercial dialyzers in Japan, with a uniform cross-sectional structure (Figure 2) in which 


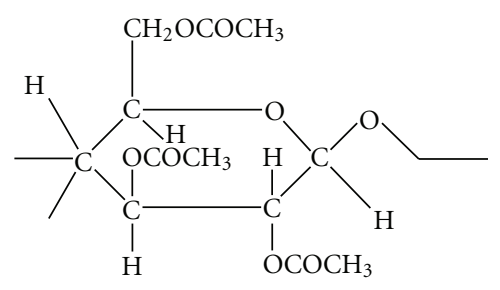

FIgure 1: The structural formula of CTA. CTA is formed by substituting three hydroxyls within cellulose.

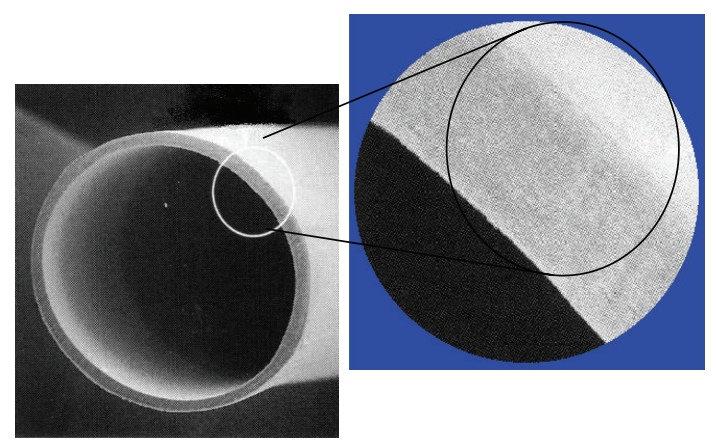

Figure 2: The scanning electron microscopy findings of crosssectional structure of CTA. The cross-sectional structure of CTA shows uniform structure in which pore size from inside (blood side) to outside (dialysate side) the hollow fiber is equal.

pore size from inside (blood side) to outside (dialysate side) the hollow fiber is equal. Compared with those of asymmetric membrane, pore size on dialysate side is relatively smaller and that on blood side is relatively larger. Therefore, it has a characteristic that endotoxin-like substances in the dialysate are hard to permeate from dialysate side.

Since hydroxyl group in cellulose is substituted by acetyl group in CTA, complement activation by hydroxyl during dialysis as well as variations in leukocyte and granulocyte elastase is infrequently observed. Hydrophilic property of hemodialysis membrane is prone to activate coagulation factor, whereas its hydrophobic property brings about strong reaction on platelet. Property of CTA has well balance of both hydrophilicity and hydrophobicity, and it has been reported to be excellent in antithrombogenicity [1].

\section{Synthetic Membrane Dialyzer}

Synthetic type membrane is hydrophobic with a property to cause thrombosis and coagulation in contact with blood. Therefore, in case of synthetic membrane, hydrophobic functional group is substituted by hydrophilic group, or acrylic acid or polyvinylpyrrolidone (PVP) is used as a hydrophilizing agent. Possible elution from membrane has been reported since the PVP is hydrosoluble [2].

3.1. Polyethersulfone (PES) Membrane. PS membrane is synthesized by polymerizing dichlorodiphenyl sulfone with bisphenol A, whereas PES is done by polymerizing dichlorodiphenyl sulfone with dihydroxydiphenyl sulfone (Figure 3).
Therefore, PES has a similar property to that of PS, but, it is considered as bisphenol A-free membrane [3]. Further, since PES characteristically has not aliphatic part which exists in PS and atomic weight ratio of sulfone group is higher than that of PS, heat resistance, mechanical resistance, and hydrophilicity are even higher. Moreover, hydrophilicity has been further enhanced by adopting PVP as a hydrophilizing agent in the process of PES membrane manufacturing. In addition, the hydrophilicity based on the PVP exerts antithrombogenicity by inhibiting protein fouling on membrane surface.

PES membrane is characterized in the cross-section structure of this membrane (Figure 4). It has an asymmetric three-layer structure having a compact layer with fine pore size on the inside and outside of the hollow fiber as well as a support layer in the central part thereof. With a membrane thickness of $40 \mu \mathrm{m}$, the mechanical strength of this membrane depends on its support layer in the central part and the molecule sieving effect of the blood is provided by the compact layer inside the hollow fiber.

Excellent biocompatibility has been reported for PSE150D (Nipro Co., Ltd., Osaka, Japan), which adopts PES membrane, without any significant change in leukocyte, platelet, C3a, and granulocyte elastase observed during dialysis therapy [4]. With equivalent or better biocompatibility compared with PS membrane, it works more excellently (Figure 5), in particular, relating to variation in platelet due to its high hydrophilicity.

\subsection{Polymethylmethacrylate (PMMA) Membrane. PMMA} membrane is produced by mixing and solving isotactic PMMA and syndiotactic PMMA which are different in steric structure. By using PMMA, it is possible to produce membrane with wide range of pore sizes by changing conditions including the blend ratio and also it is possible to produce negatively charged membrane depending on the types of additive agent to be used. It has adsorptive capacity and because of negative membrane charge, in particular, it adsorbs larger amount of basic protein.

PMMA membrane is characterized by the finding that cross-section structure of the membrane (Figure 6) has a symmetric structure having nearly homogenous microscopic pores from inner surface to outer surface of the hollow fiber. The pore sizes on the inner surface of PMMA are larger compared with those of PS membrane. Therefore, PMMA membrane has lower sieving coefficient for beta $2_{-}$ microglobulin ( $\beta 2-\mathrm{MG})$ area than that of PS membrane but it is excellent in absorptive removal of substances with molecular weight of $\beta 2-\mathrm{MG}$ and those more than 50,000 dalton, which are hard to remove by PS membrane.

PMMA membrane is known for its excellent biocompatibility based on many reports that it causes less cytokine production such as TNF- $\alpha$ and NO synthesis [5]. It has also been reported that PMMA membrane adsorbs and removes factor $\mathrm{D}$ which triggers alternative pathway complement activation [6]. In cases of acute kidney injury, it has been reported that PMMA membrane with excellent biocompatibility achieves higher survival rate compared with a case of Cuprophan (regenerated cellulose) membrane [7]. 


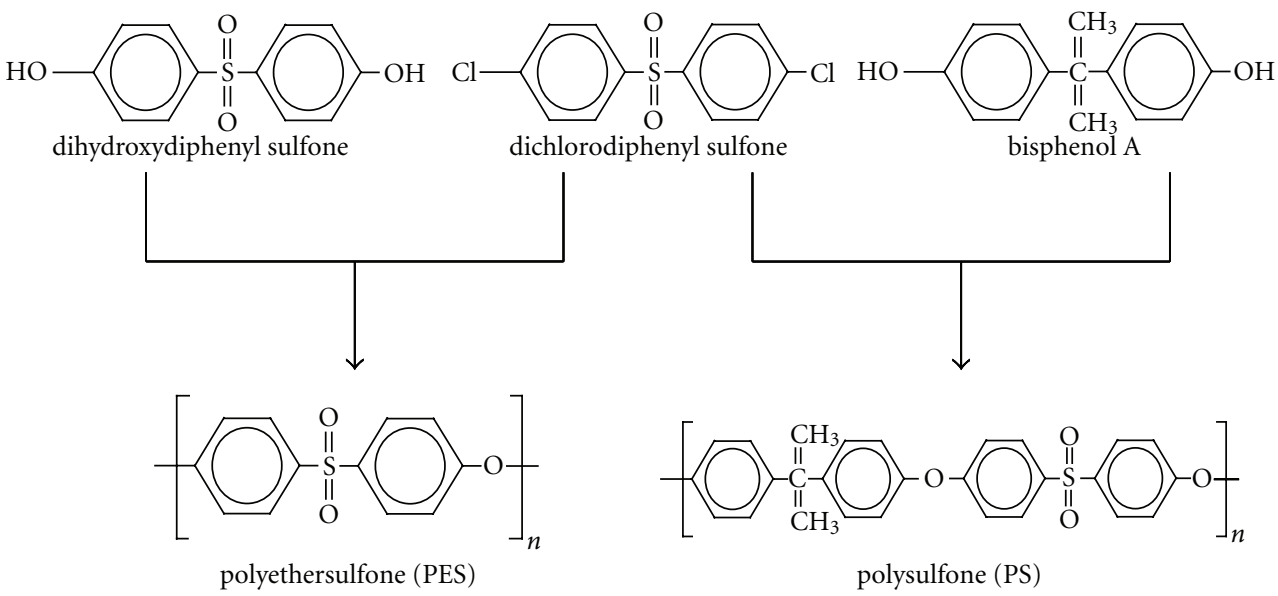

FIGURE 3: The structural formulas of PES and PS. PS membrane is synthesized by polymerizing dichlorodiphenyl sulfone with bisphenol A, whereas PES is done by polymerizing dichlorodiphenyl sulfone with dihydroxydiphenyl sulfone. Therefore, PES has similar property to PS, but risk of bisphenol A elution from membrane material is lower than that of PS.

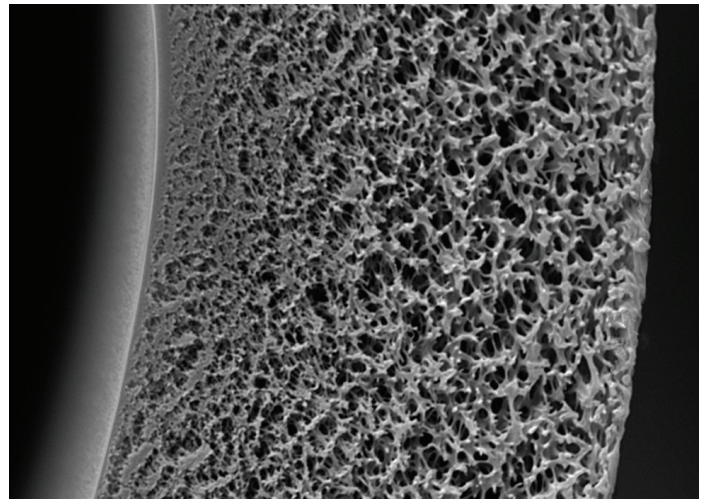

Figure 4: The scanning electron microscopy findings of crosssectional structure of PES. The cross-section structure of PES has an asymmetric three-layer structure having a compact layer with fine pore size on the inside and outside of the hollow fiber as well as a support layer in the central part thereof.

3.3. Ethylene Vinyl Alcohol (EVAL) Membrane. EVAL membrane is produced by solution polymerization between ethylene and vinyl acetate as well as alkali saponification. As chemical structural formula of EVAL membrane is expressed that vinyl alcohol group with hydrophilicity and ethylene group with hydrophobicity are combined at a certain rate, hydrophilic segment and hydrophobic segment are associated with solute permeability and mechanical strength, respectively.

Platelet-neutrophil complex formation is enhanced by the activations of both platelet and complement which are triggered when hemodialysis membrane contacts with blood. Neutrophil activation, such as radical oxygen production, is triggered by platelet-neutrophil complex [8]. Since these activations of leukocyte and platelet affect hemorheology, microcirculatory disorder could be triggered during HD. It has been reported that impact on leukocyte and platelet is lower in EVAL membrane compared with that in PS

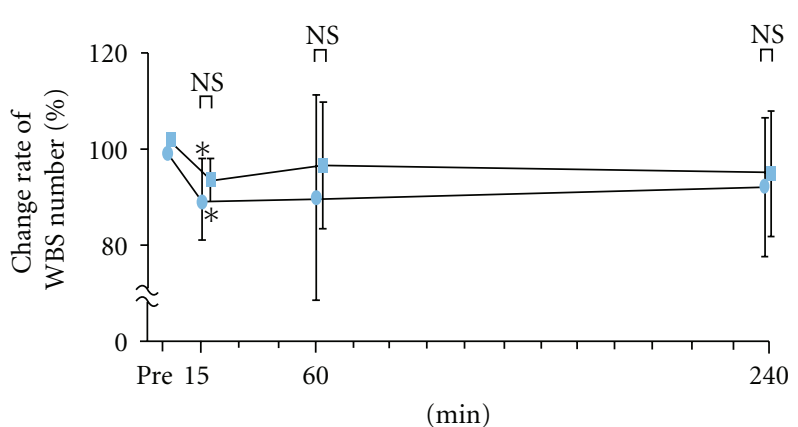

(a)

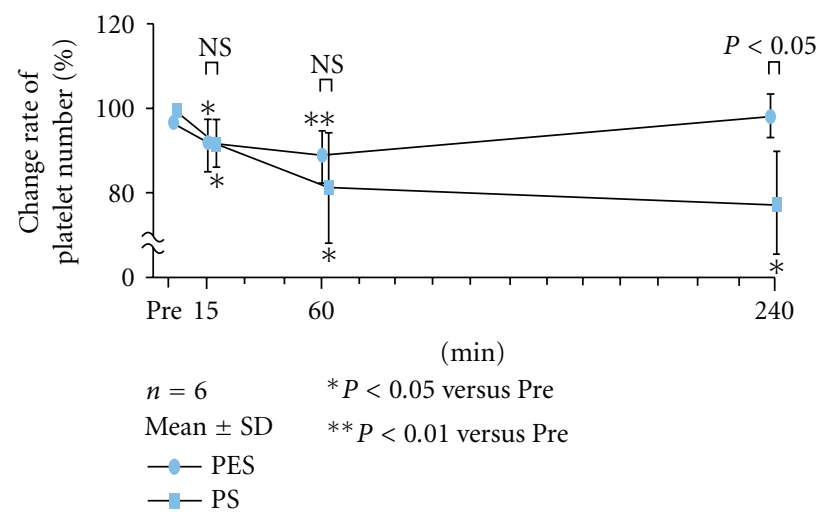

(b)

FIGURE 5: The change of WBC and platelet number during HD session by using PSE-150D (Nipro Co., Ltd., Osaka, Japan) and PS. Compared with PS, PES has excellent biocompatibility, in particular, relating to variation in platelet due to its high hydrophilicity (This figure is due to Dr. Nii's kindness.)

membrane or cellulose diacetate membrane [9]. It has also been reported that microcirculatory disorder is hard to occur during HD with EVAL membrane due to its excellent biocompatibility [10]. 


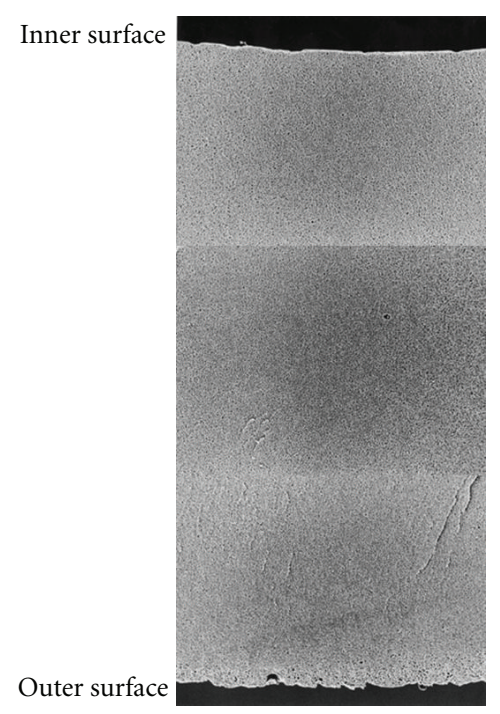

FIgURE 6: The scanning electron microscopy findings of crosssection structure of PMMA. PMMA has a symmetric structure having nearly homogenous microscopic pores from inner surface to outer surface of hollow fiber.

3.4. Vitamin E-Coated Polysulfone (PS) Membrane. During hemodialysis session, large amount of antioxidant materials is consumed in the body since the neutrophilic activation, the radical oxygen production, and the resulting oxidant stress reach excess states by the blood contact with hemodialysis membrane [11]. In order to reduce these excess states of the oxidant stress, following dialysis membrane has been developed that vitamin $\mathrm{E}$ with antioxidant effect is coated on cellulose membrane or PS membrane [12], and it has been reported that active oxygen production is suppressed and that oxidant stress is reduced during hemodialysis $[13,14]$.

\section{Other Blood Purification Devices}

In Japan, a variety of adsorption materials, which more selectively remove disease-related substances based on principle of adsorption, have been developed and applied clinically. Adsorption materials used for blood purification are roughly divided into blood adsorbent (hemoadsorption column) for direct hemoperfusion and plasma adsorbent (plasma adsorption column) for plasma perfusion.

\subsection{Hemoadsorption Column}

4.1.1. $\beta 2-M G$ Adsorption Column (Lixelle). $\beta 2-\mathrm{MG}$, which is a hydrophobic protein with molecular weight of 11,800 , is accumulated during long-term dialysis. Further, insoluble amyloid protein which consists primarily of $\beta 2-\mathrm{MG}$ with various modifications is accumulated in organs throughout the body and it results in a development of amyloidosis (dialysisrelated amyloid (DRA)). DRA causes deterioration in ADL and QOL of dialysis patients. Accumulation of $\beta 2-\mathrm{MG}$ not only leads ADL and QOL of dialysis patients to deteriorate but also adversely affects survival. Therefore, HD or HDF based on high-flux membrane has been performed aiming at

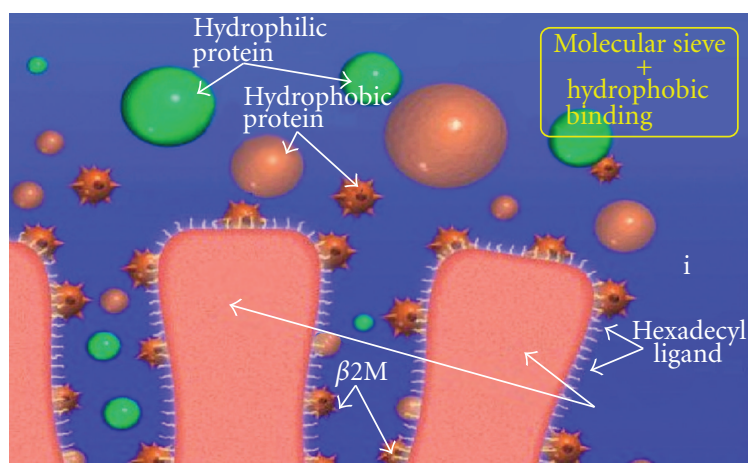

FIgURE 7: The schema of the Lixelle column. The Lixelle column is designed to adsorb $\beta 2-\mathrm{MG}$ selectively by combined use of hydrophobic interaction and adequate pore size.

removing $\beta 2-\mathrm{MG}$ from the body, and ultrapure dialysate and excellent biocompatible dialyzers have been applied aiming at inhibiting $\beta 2-\mathrm{MG}$ production. Direct hemoperfusion with $\beta 2-\mathrm{MG}$ adsorber column (Lixelle: Kaneka Co., Ltd., Osaka, Japan) was developed in 1996 which is capable of powerfully removing $\beta 2-\mathrm{MG}$ from the blood [15]. The Lixelle column contains $350 \mathrm{~mL}$ of porous cellulose adsorbent beads with a diameter of about $460 \mu \mathrm{m}$, in which a hexadecyl group with high hydrophobicity is used as ligand porous cellulose adsorbent beads. It incorporates peptides and proteins with a molecular weight of 4,000 to 20,000 dalton by the molecule sieving effect of the surface pores of the beads (Figure 7), whereas total protein, albumin, immunoglobulins, and lipids are not adsorbed [16]. Through the clinical effect of Lixelle, improvements not only in arthralgia, nocturnal awakening, and ADL but also in pinch strength and median motor terminal latency were observed after a year of Lixelle therapy $[17,18]$. In Lixelle, biocompatibility is a crucial issue since blood is directly perfused through adsorbent. As it has been reported that HD combined with Lixelle increases myeloperoxidase and polymorphonuclear leukocyte-elastase compared with HD not combined with it, further examinations of the biocompatibility are required [19].

4.1.2. Endotoxin Removal Column (Toraymyxin). A column for directly removing endotoxin from the blood (Toraymyxin: Toray Industries, Osaka, Japan) was developed for treatment of endotoxin shock which is one of the causes of prerenal acute kidney injury [20]. Toraymyxin is filled with polymyxin-B-immobilized polystyrene derivative fibers. Polymyxin B fixed on a fibrous carrier exerts a function to neutralize the activity of endotoxin by combining with lipid A, which is an active center of the activity (Figure 8). A new functional mechanism has been reported for Toraymyxin that it has an effect to adsorb endogenous cannabinoid which is an early mediator of endotoxin shock [21]. In patients with septic shock, a lot of clinical effects of Toraymyxin have been reported, including elevation in blood pressure, reduction of vasopressor, decrease in 28-day mortality and hospital mortality, and improvement in organ failure [22]. 


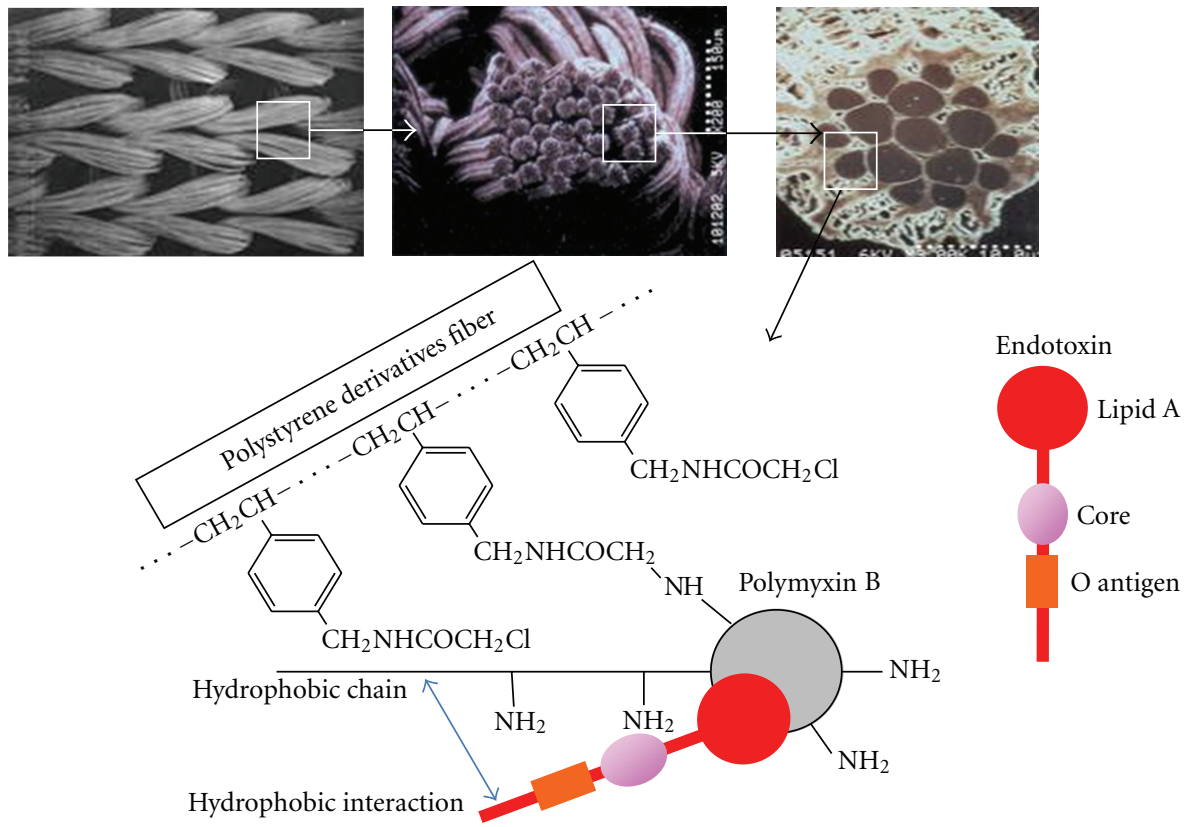

Figure 8: The schema of toraymyxin. Toraymyxin is filled with polymyxin-B-immobilized polystyrene derivative fibers. Polymyxin B fixed on a fibrous carrier exerts a function to neutralize the activity of endotoxin by combining with lipid A, which is an active center of the activity.

Even though a risk that a possibility of fixed polymyxin B is eluted cannot be totally excluded, clinically apparent side effect has not been recognized [23].

4.1.3. Leukocyte Removal Therapy (LRT). LRT is a therapy to directly remove leukocyte from the blood which is considered to be related as a cause of disease or symptoms. There are two types of therapy for the present, that is, leukocytapheresis (LCAP) and granulocytapheresis (GCAP), and they have clinical indications for inflammatory bowel disease and rheumatoid arthritis in Japan [24-27].

(1) LCAP. Polyethylene terephthalate (PET) fiber with a diameter of $3 \mu \mathrm{m}$ or less has a property to adsorb almost $100 \%$ of granulocyte and monocyte and 30 to $60 \%$ of lymphocyte and platelet. Cellsorba (Asahi Medical Co., Tokyo, Japan) is comprised of a rolled nonwoven fabric of PET fiber with a fiber diameter ranging from 0.8 to $2.8 \mu \mathrm{m}$ for selectively removing leukocyte.

Problems of biocompatibility on PET include bradykinin shock which occurs in patients who are orally taking angiotensin-converting enzyme (ACE) inhibitor since PET is negatively charged. Blood coagulation system is strongly activated by the blood contacts with a negatively charged material, and it results in production of bradykinin (BK) having vasodilating action. This $\mathrm{BK}$ is inactivated by kininase II which is an enzyme identical to ACE. Therefore, the function of kininase II which is an enzyme identical to ACE is inhibited by taking ACE inhibitor and it causes increase in BK concentration in the blood, vasodilatation, and shock symptom (Figure 9). Besides, some reactions on the skin of blood return, such as pain, redness, and swelling, could be brought by various chemical mediators which are released from active leukocyte and platelet adsorbed within Cellsorba.

(2) GCAP. Cellulose acetate (CA) beads combine with immunoglobulin (IgG) and complement active C3b/C3bi. Granulocyte and monocyte have receptors for Fc of IgG or complement and are selectively trapped via immunoglobulin and complement which are combined with CA and these receptors.

Adacolumn (JIMRO, Takasaki, Japan) is a purifier filled with 30,000 of CA beads with a diameter of $3 \mathrm{~mm}$ for selectively removing granulocyte and monocyte. During the purification, neither lymphocyte nor platelet is removed. The complications, which included venous pressure elevation, venous access difficulty, coagulation in blood circuit, and difficulty in returning blood, occurred during 2.3\% of GCAP. However, there were no serious adverse events [28].

\subsection{Plasma Adsorption Column}

4.2.1. Plasma Adsorption Based on a Principle of Hydrophobic Bonding (Immusorba PH-350/TR-350). Autoantibody and immune complex have hydrophobic group. Both phenylalanine and tryptophan are hydrophobic amino acids widely distributed in the body. Immusorba PH-350 (Asahi Medical Co., Tokyo, Japan) is using phenylalanine as a ligand, and TR350 (Asahi Medical Co., Tokyo, Japan) is using tryptophan as a ligand. These columns covalently bond with the hydroxyl group on the surface of porous polyvinyl alcohol gel with the amino group of amino acid (Figure 10). These columns could selectively remove autoantibody or immune complex 


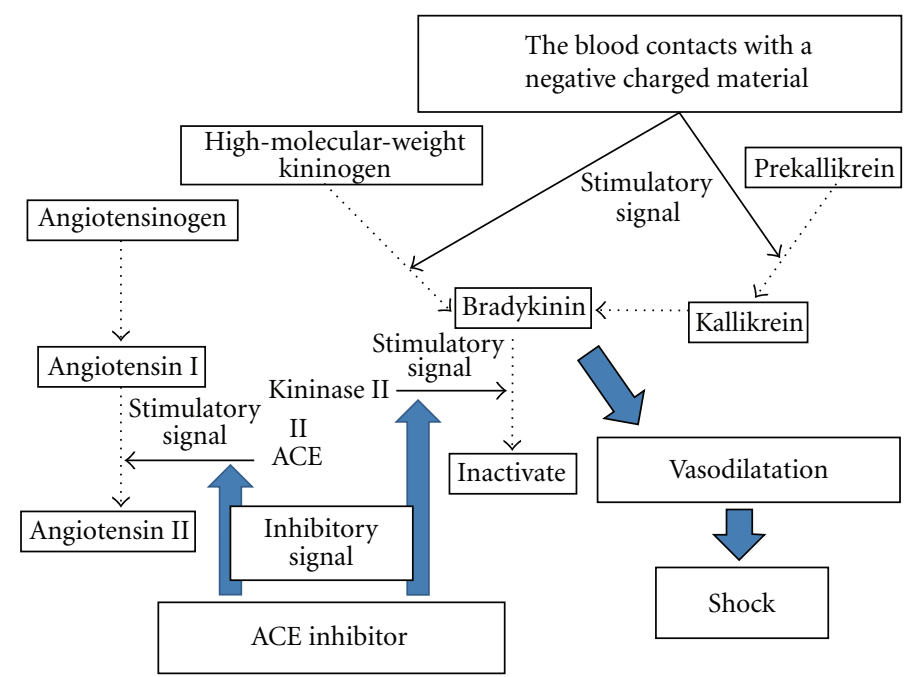

FIGURE 9: The mechanism of bradykinin-induced anaphylactic shock. When the blood contacts with a negatively charged material, it results in production of Bradykinin (BK) having vasodilating action. The bioactivity of this BK is inactivated by kininase II which is an enzyme identical to ACE. Therefore, the function of kininase II which is an enzyme identical to ACE is inhibited by taking ACE inhibitor and it causes increase in BK concentration in the blood, vasodilatation, and shock symptom being unable to inactivate BK.

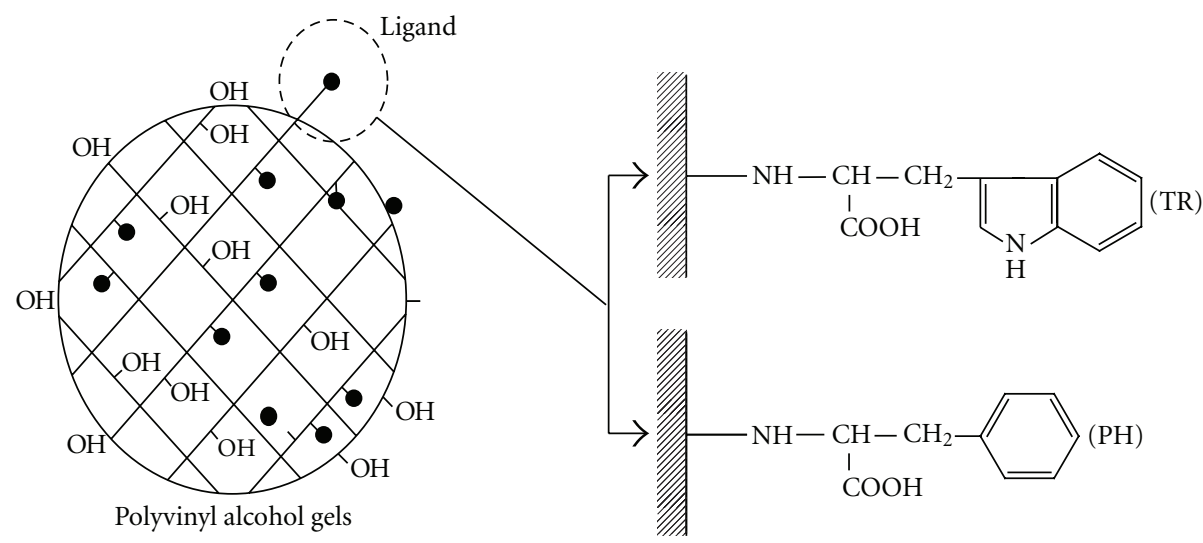

Figure 10: The structural formulas of Immusorba PH-350 and TR-350. These columns covalently bond with the hydroxyl group on the surface of porous polyvinyl alcohol gel with the amino group of amino acid. Immusorba $\mathrm{PH}-350$ is using phenylalanine as ligand and TR-350 is using tryptophan.

by hydrophobic bonding. Further, as these adsorbing materials are charged negatively, the affinity between ligand of these columns and antibody was mainly hydrophobic interaction and was partially caused by the ionic interaction based on carboxylic group (Figure 11). The clinical effects of these columns have been reported on lupus nephritis, myasthenia gravis, multiple sclerosis, and other collagen diseases [29-33].

As both Immusorba PH-350 and TR-350 are charged negatively, shock symptom is triggered by bradykinin in patients who are taking ACE inhibitor. In addition, reduction in $\mathrm{Ca}$ ion level could be observed temporarily during treatment (Figure 12) because the positive ion could be absorbed by the negative charge.

4.2.2. Plasma Adsorption Based on Electrostatic Bond (Liposorber, Selesorb). Apo-B, anti-DNA antibody, immune com- plex, or antigen-recognizing site on anticardiolipin antibody is charged positively. Liposorber (Kaneka Co., Ltd., Osaka, Japan) and Selesorb (Kaneka Co., Ltd., Osaka, Japan) are a column, which includes cellulose beads with the negative charge of dextran sulphate. The affinity between negative charge of these columns and these positive-charged etiological substances was mainly caused by the ionic interaction (Figure 13). Alternatively, Selesorb is devised to enhance selectivity of anti-DNA antibody, immune complex, and anticardiolipin antibody which are smaller than apo-B by making pore structure of cellulose gel smaller (Figure 14). As both Liposorber and Selesorb are charged negatively, they have a risk of developing shock symptom by bradykinin in patients who are taking ACE inhibitor.

(1) Liposorber. LDL cholesterol level is drastically reduced by 60 to $80 \%$ by single LDL apheresis using Liposorber, 


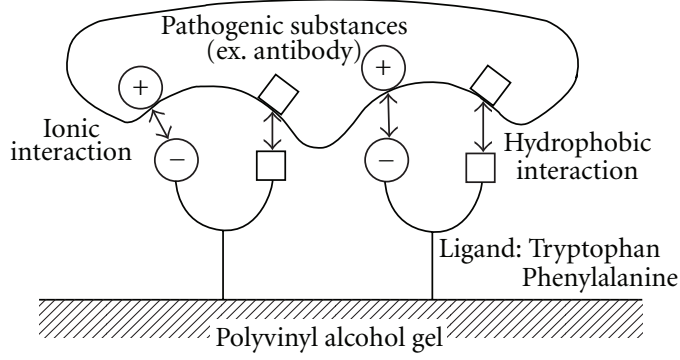

FIgURE 11: The schema of Immusorba PH-350 and TR-350. The affinity between ligand of these columns and antibody was mainly hydrophobic interaction and partially caused by the ionic interaction based on carboxylic group.

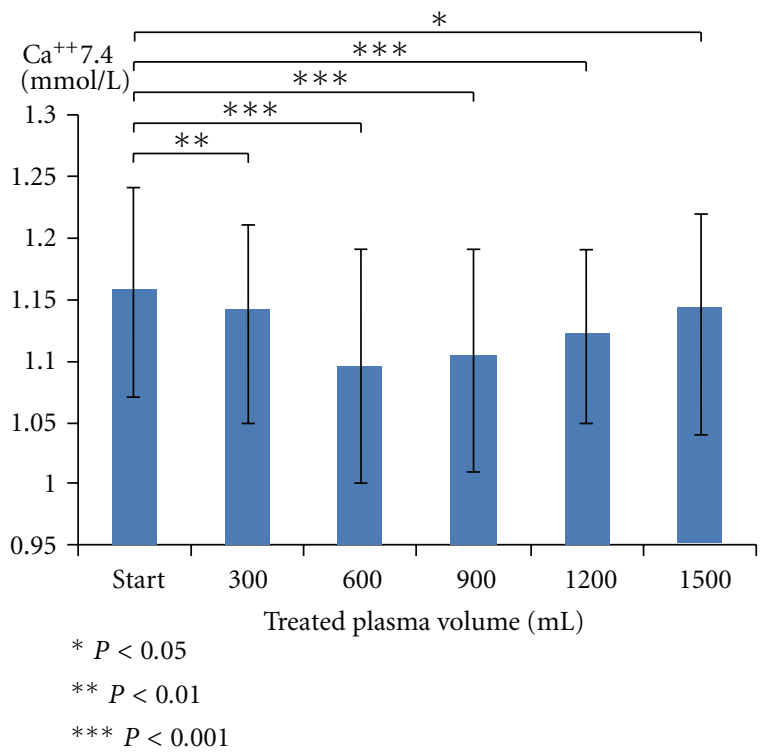

FIgure 12: The change of serum Ca ion level during immunoadsorption with Immusorba TR-350. Reduction of Ca ion level could be observed during treatment by using Immusorba TR-350, because positive ion could be absorbed by the negative charge.

whereas HDL cholesterol level is hardly reduced. LDL apheresis using Liposorber is conducted for the heterofamilial hyperlipidemic patients, and clinical effects have been reported that incidence of cardiovascular event reduced by $70 \%$ compared with those in groups treated based on medication alone [34]. Further, the clinical effects of LDL apheresis using Liposorber have been reported also on arteriosclerosis obliterans for which hyperlipidemia is one of the risk factors [35]. The mechanisms are supposed to have impacts on (i) improvement in viscosity of blood and plasma, (ii) improvement in erythrocyte deformability, (iii) increase of vasodilator such as bradykinin and NO, (ix) reduction in coagulation factor such as fibrinogen, (x) reduction in cell adhesion factor, (xi) inhibition of platelet activation, and (xii) inhibition of oxidizability of LDL. Moreover, the progression of renal dysfunction due to focal glomerulosclerosis (FGS) which presents refractory

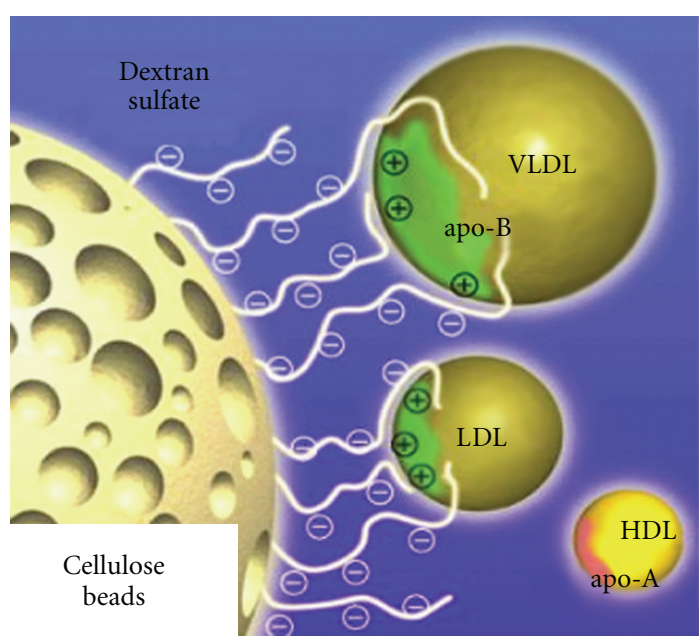

Liposorber

(a)

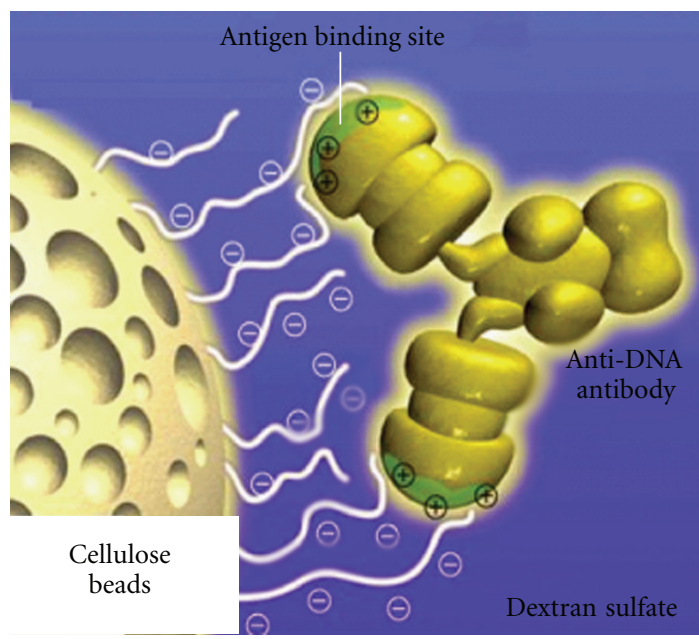

Selesorb

(b)

Figure 13: The schema of the Liposorber and Selesorb. Apo$\mathrm{B}$, anti-DNA antibody, immune complex, or antigen-recognizing site on anticardiolipin antibody is charged with positive charge. Liposorber and Selesorb include cellulose beads with the negative charge of dextran sulphate. The affinity between negative charge of these columns and these positive charged etiological substances was mainly caused by the ionic interaction.

nephrotic syndrome to steroids was ameliorated by LDL apheresis. Glomerular sclerosis is thought to be caused by systemic or intraglomerular hypertension associated with arteriosclerosis progress, enhanced cytokine secretion as well as mesangial cell proliferation associated with increase of macrophage in glomerulus, and disorder of mesangial cell, and they are associated with increase of LDL in the blood [36].

In a multi-institutional study of LDL apheresis using Liposorber for steroid-resistant nephrotic syndrome, significant reduction in urine protein and elevation in serum 


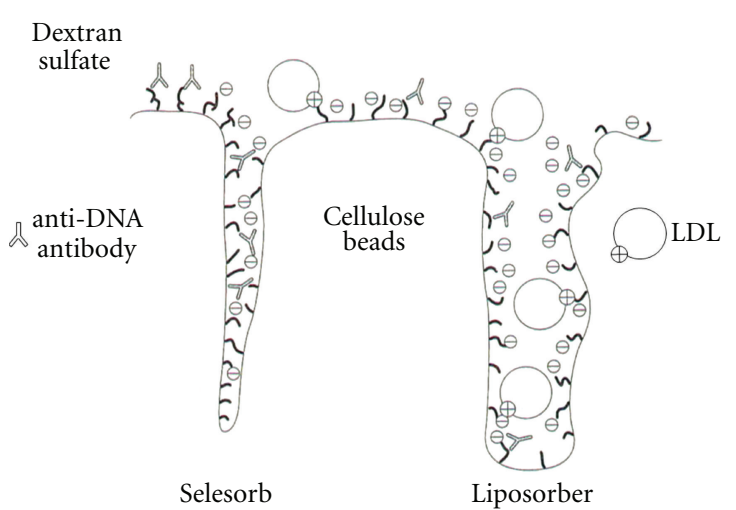

Figure 14: The difference of between Liposorber and Selesorb. The pore size of Selesorb beads is smaller than that of Liposorber so that causative antibodies including anti-DNA antibody are selectively adsorbed avoiding larger-size molecules such as LDL.

albumin level were recognized which shortened the days required for reducing urine protein level to less than $3.5 \mathrm{~g} /$ day. In addition, the following clinical effects have been also reported that remission rate in two years after completion of treatment is significantly high [37]. This paper pointed out the following factors for possible mechanisms of LDL apheresis using Liposorber: improvement of hyperlipidemia as well as inhibition of hypercoagulation, correction of disorder of renal lipid mediator (thromboxane A2 hyperproduction state), inhibition of infiltration of macrophage to glomerulus based on inhibitory action on cytokine production, restoration of steroid-responsiveness, and enhancement of intracellular incorporation of cocurrent use of cyclosporine.

(2) Selesorb. By inmmunoabsorption therapy with Selesorb, the mean removal rate of anti-DNA antibody was $34 \%$, moreover, anticardiolipin antibody and immune complex were efficiently removed. The clinical features of systemic lupus erythematosus, such as facial erythema and hematologic disorder, were also improved without severe adverse effects [38].

\section{Conclusion}

It is important that blood purification therapy should be performed consistently evaluating not only risks associated with these bioincompatibilities but also loads to cardiovascular system posed by extracorporeal circulation, risks relating to vascular access creation, and exacerbation of hemorrhagic tendency caused by anticoagulant, together with advantages obtained from treatments. The characteristics of biocompatibility of each material mentioned here will bring important information for safer blood purification therapy.

\section{Acknowledgment}

The authors thank Dr. Nii for quoting his data.

\section{References}

[1] K. Kumei, Y. Futoo, K. Inoue, Y. Tajima, and S. Yokose, "Applicability of HPM dialyzers to the treatment of diabetic nephropathy (antithrombogenicity)," Japanese Society for Dialysis Therapy, vol. 29, pp. 1529-1537, 1994 (Japanese).

[2] M. Matsuda, M. Sato, H. Sakata et al., "Effects of fluid flow on elution of hydrophilic modifier from dialysis membrane surfaces," Journal of Artificial Organs, vol. 11, no. 3, pp. 148155, 2008.

[3] H. Yamasaki, Y. Nagake, and H. Makino, "Determination of bisphenol A in effluents of hemodialyzers," Nephron, vol. 88, no. 4, pp. 376-378, 2001.

[4] H. Naitoh, M. Agazuma, M. Suzuki, and I. Aoike, "Clinical evaluation of hollow fiber type hemodialyzer PES-150D," Research of New Medical Device, vol. 4, no. 2, pp. 15-24, 1998 (Japanese).

[5] A. Amore, R. Bonaudo, D. Ghigo et al., "Enhanced production of nitric oxide by blood-dialysis membrane interaction," Journal of the American Society of Nephrology, vol. 6, no. 4, pp. 1278-1283, 1995.

[6] M. Pascual, J. A. Schifferli, J. G. Pannatier, and J. P. Wauters, "Removal of complement factor D by adsorption on polymethylmethacrylate dialysis membranes," Nephrology Dialysis Transplantation, vol. 8, no. 11, pp. 1305-1306, 1993.

[7] R. M. Hakim, R. L. Wingard, and R. A. Parker, "Effect of the dialysis membrane in the treatment of patients with acute renal failure," New England Journal of Medicine, vol. 331, no. 20, pp. 1338-1342, 1994.

[8] S. Itoh, K. Takeshita, C. Susuki, K. Shige-eda, and T. Tsuji, "Redistribution of P-selectin ligands on neutrophil cell membranes and the formation of platelet-neutrophil complex induced by hemodialysis membranes," Biomaterials, vol. 29, no. 21, pp. 3084-3090, 2008.

[9] V. Sirolli, E. Ballone, S. di Stante, L. Amoroso, and M. Bonomini, "Cell activation and cellular-cellular interactions during hemodialysis: effect of dialyzer membrane," International Journal of Artificial Organs, vol. 25, no. 6, pp. 529-537, 2002.

[10] M. Sato, H. Morita, H. Ema, S. Yamaguchi, and I. Amano, "Effect of different dialyzer membranes on cutaneous microcirculation during hemodialysis," Clinical Nephrology, vol. 66, no. 6, pp. 426-432, 2006.

[11] J. Himmelfarb, "Oxidative stress in hemodialysis. In: Ronco C, Cruz DN, (eds): hemodialysis - from basic research to clinical trials," Contributions to Nephrology, vol. 161, pp. 132-137, 2008.

[12] M. Sasaki, N. Hosoya, and M. Saruhashi, "Vitamin E modified cellulose membrane," Artificial Organs, vol. 24, no. 10, pp. 779-789, 2000.

[13] M. Satoh, Y. Yamasaki, Y. Nagake et al., "Oxidative stress is reduced by the long-term use of vitamin E-coated dialysis filters," Kidney International, vol. 59, no. 5, pp. 1943-1950, 2001.

[14] D. C. Tarng, T. P. Huang, T. Y. Liu, H. W. Chen, Y. J. Sung, and Y. H. Wei, "Effect of vitamin E-bonded membrane on the 8-hydroxy 2'-deoxyguanosine level in leukocyte DNA of hemodialysis patients," Kidney International, vol. 58, no. 2, pp. 790-799, 2000.

[15] S. Furuyoshi, M. Nakatani, J. Taman, H. Kutsuki, S. Takata, and N. Tani, "New adsorption column (Lixelle) to eliminate $\beta_{2}$-microglobulin for direct hemoperfusion," Therapeutic Apheresis, vol. 2, no. 1, pp. 13-17, 1998. 
[16] M. Nakaya, S. Furuyoshi, S. Takata, and N. Tani, "Adosrption characteristics of adsorbent column (Lixelle) for the treatment of dialysis-related amyloidosis," Japanese Journal for Artificial Organs, vol. 27, pp. 571-577, 1988 (Japanese).

[17] F. Gejyo, Y. Kawaguchi, S. Hara et al., "Arresting dialysisrelated amyloidosis: a prospective multicenter controlled trial of direct hemoperfusion with a $\beta_{2}$-microglobulin adsorption column," Artificial Organs, vol. 28, no. 4, pp. 371-380, 2004.

[18] T. Abe, K. Uchita, H. Orita et al., "Effect of $\beta_{2}$-microglobulin adsorption column on dialysis-related amyloidosis," Kidney International, vol. 64, no. 4, pp. 1522-1528, 2003.

[19] K. Inose, K. Ono, Y. Okubo, R. Yoshida, and T. Naruse, "The elevation of plasma levels of myeloperoxidase and polymorphonuclear leukocyte elastase as an index of bioincompatibility of the column during hemodialysis using with a $\beta_{2}$-microglobulin-selective adsorbent column," Clinical and Experimental Nephrology, vol. 4, no. 1, pp. 52-57, 2000.

[20] H. Shoji, T. Tani, K. Hanasawa, and M. Kodama, "Extracorporeal endotoxin removal by polymyxin B immobilized fiber cartridge: designing and antiendotoxin efficacy in the clinical application," Therapeutic Apheresis, vol. 2, no. 1, pp. 3-12, 1998.

[21] T. Sato, H. Shoji, and N. Koga, "Endotoxin adsorption by polymyxin B immobilized fiber column in patients with systemic inflammatory response syndrome: the Japanese experience," Therapeutic Apheresis, vol. 7, no. 2, pp. 252-258, 2003.

[22] D. N. Cruz, M. Antonelli, R. Fumagalli et al., "Early use of polymyxin B hemoperfusion in abdominal septic shock: the EUPHAS randomized controlled trial," JAMA-Journal of the American Medical Association, vol. 301, no. 23, pp. 2445-2452, 2009.

[23] H. Suzuki, H. Nemoto, H. Nakamoto et al., "Continuous hemodiafiltration with polymyxin-B immobilized fiber is effective in patients with sepsis syndrome and acute renal failue," Therapeutic Apheresis, vol. 6, no. 3, pp. 234-240, 2002.

[24] A. Sakuraba, S. Motoya, K. Watanabe et al., "An open-label prospective randomized multicenter study shows very rapid remission of ulcerative colitis by intensive granulocyte and monocyte adsorptive apheresis as compared with routine weekly treatment," American Journal of Gastroenterology, vol. 104, no. 12, pp. 2990-2995, 2009.

[25] Y. Fukuda, T. Matsui, Y. Suzuki et al., "Adsorptive granulocyte and monocyte apheresis for refractory Crohn's disease: an open multicenter prospective study," Journal of Gastroenterology, vol. 39, no. 12, pp. 1158-1164, 2004.

[26] K. Mitsuyama, H. Yamasaki, K. Kuwaki, H. Takedatsu, and M. Sata, "Recent understanding of leukocytapheresis (LCAP) for the treatment of inflammatory bowel disease," Current Pharmaceutical Design, vol. 15, no. 18, pp. 2110-2119, 2009.

[27] Y. Ueki, A. Sagawa, K. Tanimura et al., "A multicenter study of leukocytapheresis in rheumatoid arthritis," Clinical and Experimental Rheumatology, vol. 25, no. 6, pp. 810-816, 2007.

[28] T. Hibi, Y. Sameshima, Y. Sekiguchi et al., "Treating ulcerative colitis by Adacolumn therapeutic leucocytapheresis: clinical efficacy and safety based on surveillance of 656 patients in 53 centres in Japan," Digestive and Liver Disease, vol. 41, no. 8, pp. 570-577, 2009.

[29] K. Sugimoto, K. Yamaji, K. S. Yang, Y. Kanai, H. Tsuda, and H. Hashimoto, "Immunoadsorption plasmapheresis using a phenylalanine column as an effective treatment for lupus nephritis," Therapeutic Apheresis and Dialysis, vol. 10, no. 2, pp. 187-192, 2006.
[30] E. Schmitt, K. Appen, E. Behm et al., "Immunoadsorption with phenylalanine-immobilized polyvinyl alcohol versus plasma exchange, a controlled pilot study in multiple sclerosis," in Therapeutic Plasmaphersis (XII), T. Agishi, A. Kawamura, M. Mineshima, and T. Agishi, Eds., pp. 779-783, VSP International Science Publisher, Utrecht, The Netherlands, 1993.

[31] S. Okamiya, M. Ogino, Y. Ogino et al., "Tryptophanimmobilized column-based immunoadsorption as the choice method for plasmapheresis in Guillain-Barré syndrome," Therapeutic Apheresis and Dialysis, vol. 8, no. 3, pp. 248-253, 2004.

[32] K. Miyamoto and S. Kusunoki, "Intermittent plasmapheresis prevents recurrence in neuromyelitis optica," Therapeutic Apheresis and Dialysis, vol. 13, no. 6, pp. 505-508, 2009.

[33] D. Grob, D. Simpson, H. Mitsumoto et al., "Treatment of myasthenia gravis by immunoadsorption of plasma," Neurology, vol. 45, no. 2, pp. 338-344, 1995.

[34] H. Mabuchi, J. Koizumi, M. Shimizu et al., "Long-term efficacy of low-density lipoprotein apheresis on coronary heart disease in familial hypercholesterolemia," American Journal of Cardiology, vol. 82, no. 12, pp. 1489-1495, 1998.

[35] T. Agishi, Y. Kitano, T. Suzuki et al., "Improvement of peripheral circulation by low density lipoprotein adsorption," American Society for Artificial Internal Organs Transactions, vol. 35, no. 3, pp. 349-351, 1989.

[36] J. F. Moorhead, M. K. Chan, M. El-Nahas, and Z. Varghese, "Lipid nephrotoxicity in chronic progressive glomerular and tubulo-interstitial disease," The Lancet, vol. 2, no. 8311, pp. 1309-1311, 1982.

[37] E. Muso, M. Mune, Y. Fujii et al., "Significantly rapid relief from steroid-resistant nephrotic syndrome by LDL apheresis compared with steroid monotherapy," Nephron, vol. 89, no. 4, pp. 408-415, 2001.

[38] K. Suzuki, M. Kawagoe, M. Hara et al., "Clinical evaluation of immunoadsorption in patients with systemic lupus erythematosus using two dextran sulfate cellulose columns (SL01, SelesorbTM) in an automated column regeneration unit," Recenti Progressi in Medicina, vol. 11, pp. 3007-3021, 1991 (Japanese). 

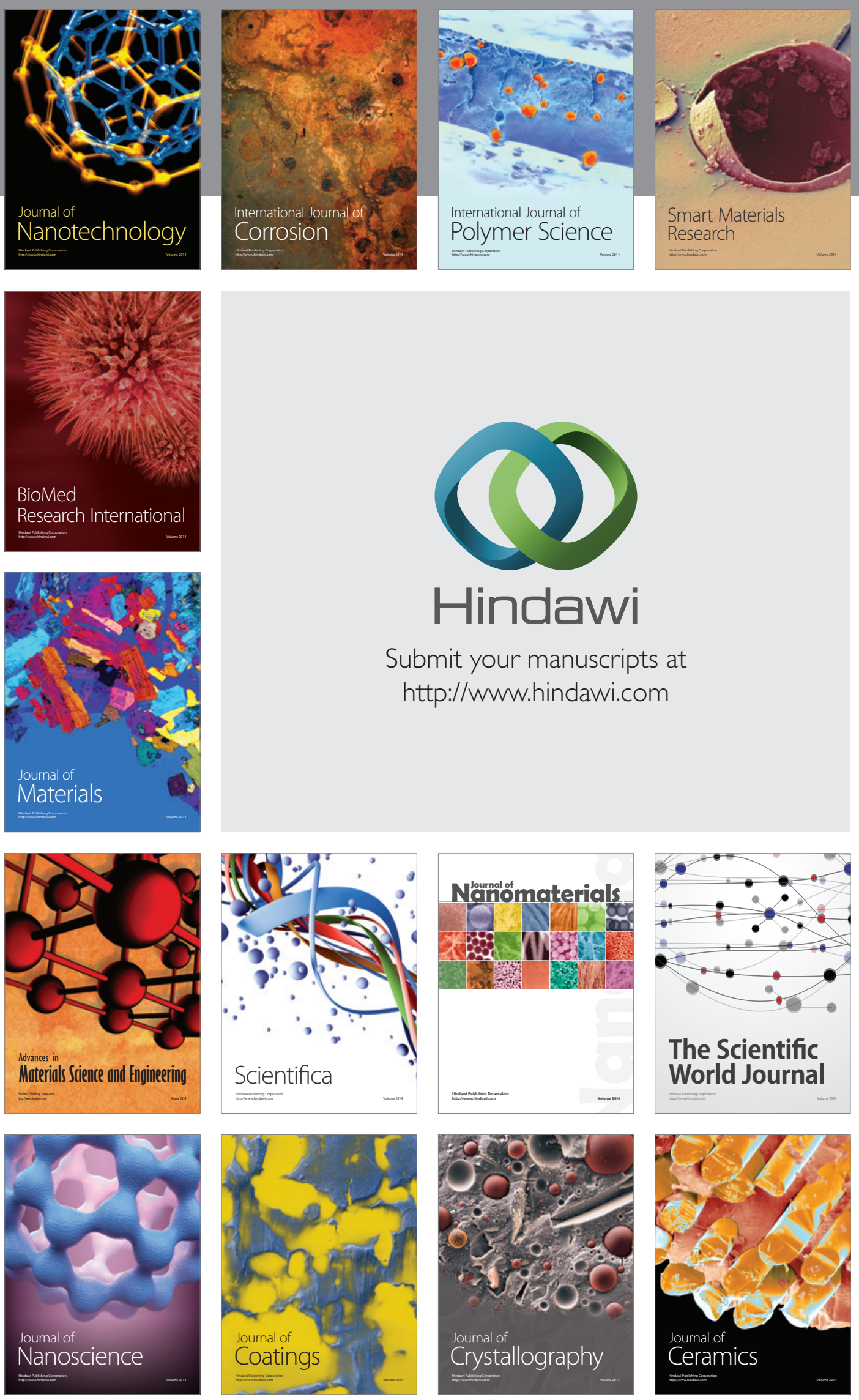

The Scientific World Journal

Submit your manuscripts at

http://www.hindawi.com

\section{World Journal}

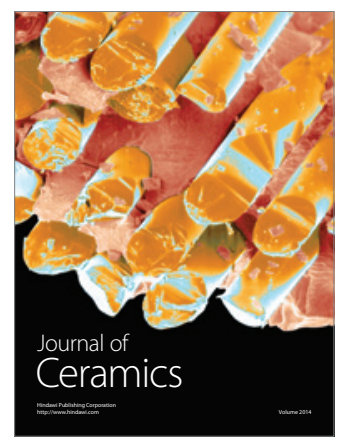

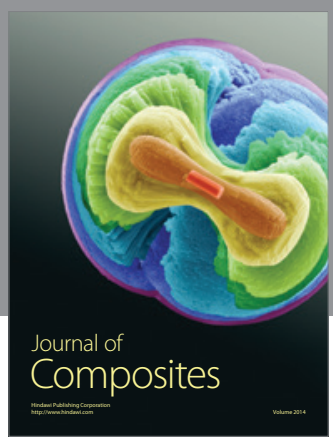
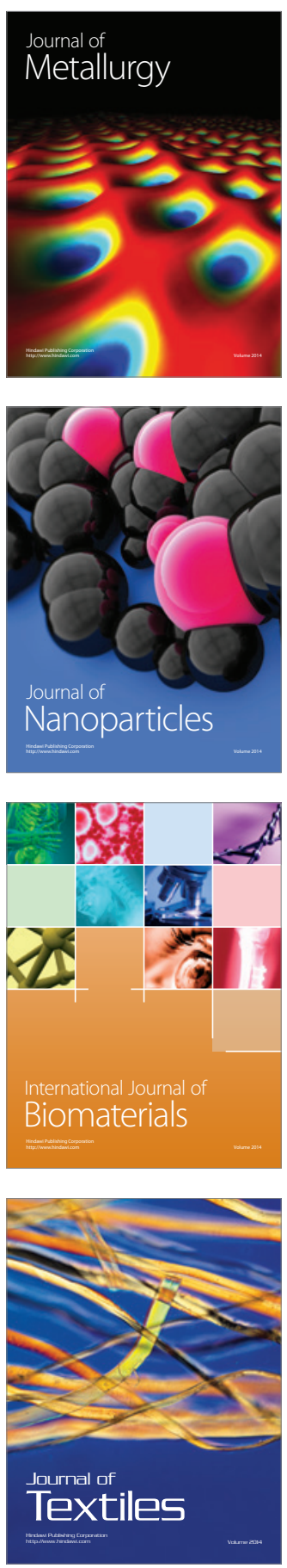